Please quote as: Prinz, A.; Menschner, P. \& Leimeister, J. M. (2012): NFC-based Electronic Data Capture Systems - The Case of a Quality of Life Questionnaire. In: Proceedings of the 20th European Conference on Information Systems (ECIS), Barcelona, Spain. 


\title{
NFC-BASED ELECTRONIC DATA CAPTURE SYSTEMS - THE CASE OF A QUALITY OF LIFE QUESTIONNAIRE
}

Prinz, Andreas, Information Systems, Kassel University, Pfannkuchstraße 1, 34121 Kassel, Germany,prinz@uni-kassel.de

Menschner, Philipp, Information Systems, Kassel University, Pfannkuchstraße 1, 34121 Kassel, Germany, menschner@uni-kassel.de

Leimeister, Jan Marco, Information Systems, Kassel University, Pfannkuchstraße 1, 34121 Kassel, Germany, leimeister@uni-kassel.de

\begin{abstract}
In this paper, we present a Near Field Communication (NFC)-based electronic data capture prototype for patient self-reported rating scales. Such scales are valuable feedback for medical treatment and care processes. As traditional paper-based questionnaires are time-and cost-consuming and may be affected by low patient compliance, our prototype allows patient monitoring and electronic data acquisition directly from the patient's home. It enables real time representation and analysis of patient data and thus allows direct medical intervention by physicians. In developing the prototype, we followed a design science approach, developed design goals for the special case of patients suffering from impaired motor skills, and tested the prototype in a field study over the course of twelve weeks. We chose NFC since the interaction paradigm is intuitive and quickly learned, without prior knowledge being necessary. Our results indicate that NFC is almost as simple as filling out a paperbased questionnaire. During the study patients used the prototype autonomously and with minimal errors. Further, NFC technology was perceived as very intuitive and the information quality of each patient's health status could be improved. Based on the findings we derive recommendations for future research and applications of NFC based electronic data capture systems.
\end{abstract}

Keywords: Electronic Data Capture, NFC, Information Quality, Design Science. 


\section{Introduction}

Patients suffering from chronic diseases often have to cope with limitations and a reduced quality of life. This is particularly applicable for patients with limited or impaired fine motor skills, which can occur from chronic diseases such as motor neuron disease, dementia, Parkinson's disease or multiple sclerosis. For most diseases of this kind, a cure remains elusive, despite availability of certain medications to treat symptoms. The protracted course of the diseases has a major impact on the quality of life of patients and their families (Welsh et al., 2003, Löser et al., 2007). In order to guarantee the best possible treatment procedures, physicians need up-to-date information about their patients' actual state and well-being. Yet, it is well recognized that capturing, archiving, analysing and interpreting health status information involves logistic challenges (Wolfe and Pincus, 1995). For patients living at home, this implies an even greater challenge, as contemporaneous information, which can be efficiently and effectively used for therapeutic decision-making, is not available. The insufficient information logistics amongst physicians, patients and nursing staff concerning the well-being of patients could be improved by using mobile networks and information systems for electronic data capture (EDC) of health status information. As mobile devices have achieved a high level of public acceptance, they are increasingly considered to be integrated in health care (Leimeister et al., 2005). Telecommunication networks allow a flexible, location-independent monitoring of the patients' status. Additionally, costs can be reduced as patients take over data acquisition tasks.

In this paper, we describe the development and evaluation of a mobile Near Field Communication (NFC)-based electronic data capture (EDC) system for patient self-reported questionnaires. NFC, is a short-range high frequency wireless communication technology, based on the frequency of 13,56 $\mathrm{MHz}$, which enables the exchange of data between devices within 10 centimetres (around 4 inches) distance (ECMA-340, 2004, Want, 2006). Therefore, the objective of our work was to provide a simple, effective and efficient self-reporting system of the current well-being status, which would allow patients a cost-efficient and easy-to-handle reporting system of their current condition. We have identified a scenario that can benefit from applying EDC techniques, both from an economic perspective as well as having an impact on the patients' health status and quality of life. These are rating scales and disease progress questionnaires.

The paper is structured as follows. We first provide an overview of related work on NFC technology and EDC methods used in patient care, clinical trials, and Ambient Assisted Living (AAL) scenarios. This is followed by a presentation of the design goals that guided our development and our research approach. Then, we shortly describe our inSERT (Self-Reporting questionnaire) prototype system and present the results of the field test evaluation. This includes a discussion and insights into the design of contactless electronic capture of self-reported patient data. We close with a discussion of the contributions from this research, the implications and the derived recommendations for future research.

\section{Related Work}

In health care, the provision of appropriate treatment often depends on self-reported states, characteristics and behaviours of patients in order to understand disease progression or treatment outcomes. Over the last decades, there has been steady progress in the development of measurement techniques for all kinds of diseases. A wide variety of rating scales is used to determine the state of patients, ranging from impairment scales to health-related quality of life instruments (e.g., (Kaufmann et al., 2005, Grauer and Birnbom, 1975, Welsh et al., 2003)). Studies have shown their importance in measuring the impact of different diseases and their treatment on patients (Guyatt et al., 1993). The results of the rating scales and questionnaires are then used to adapt and optimize therapeutic options. Several studies have evaluated the effectiveness of patient self-rating for different kinds of diseases (Strömgren et al., 2001). They show that the validity and reliability are of a sufficient level in order to serve as a ba- 
sis for treatment decision-making. Follow-up costs of inadequate treatment are hard to measure, and the negative impact on patients' quality of life is manifest.

Health status information has traditionally been captured using paper-based questionnaires. However, traditional paper-based medical patient surveys or questionnaires for health status information are time- and cost-consuming, and can suffer from contaminating effects, such as time-delay or inaccuracy due to disregard, which can lead to unsuited or out-of-date treatment. Electronic-based questionnaires overcome these deficits by allowing to aggregate and calculate the data, which potentially reduces the time to acquire data and can hence increase the patient's compliance (Nyholm et al., 2004). Further, electronically captured, self-reported patient data can instantly be compiled and can be available for physicians and clinical personnel in almost real time. This provides indications for possible medical interventions, even in-between appointments (Velikova et al., 1999).

Currently, there are considerable studies on various occurrences of electronic data capture (EDC) (Bischoff-Ferrari HA. et al., 2005, Richter et al., 2006, Palmblad M. and B., 2004, Dale O. and KB., 2007, Koene et al., 2010, Blake, 2008). The application of NFC technology to provide EDC for patient self-reporting is, however, comparatively novel and not widely implemented. NFC makes many tasks easier and more convenient by providing access to systems and services simply by touch. Morak et al. (2007), use NFC-technology as a self-management process for monitoring heart failure patients and Iglesias et al. (2009) describe an NFC-based health monitoring system to improve quality of life for elderly patients. Patients transmit health related data to a central database by touching medical devices with a mobile telephone. Physicians or nurses can view the entire data and guide the patient to the best possible health status. Bravo et al. (2008) use NFC for supporting nurse activities in an Alzheimer's day centre. NFC-based nutrition management for elderly patients is suggested by Prinz et al. (2009). In a clinical context, NFC is used by various researchers. Lahtela et al. (2008) have developed an NFCbased solution to avoid medication errors in hospitals. As an additional path of medical data acquisition, Fikry et al. (2006) and Morak et al. (2009) describe different NFC-based solutions which allow physicians or nurses to collect data by easily touching medical devices with a mobile phone. Yet, none of these studies focus on usability for patients suffering from impaired fine motor skills.

\section{Development and Research Approach}

For the specific use case we worked in cooperation with a German hospital which is specialized on amyotrophic lateral sclerosis (ALS), which is a form of motor neuron disease. ALS is a progressive, fatal, neurodegenerative disease caused by the degeneration of motor neurons, the nerve cells in the central nervous system that control voluntary muscle movement. Death usually occurs within two to five years of diagnosis. In particular, ALS patients suffer from progressive paralysis, so they need to be provided with an easy to handle system. As patients attribute an important role to the interactive applications interface design, the overall process of our approach to user-centered design (UCD) is based on evaluating the usability of inSERT in small iterative steps.

\subsection{Design Goals}

The design goals have been identified in discussions and observations with physicians and patients and formed the basis for developing our solution idea for an electronic data capture system. In this specific case we had several challenges. As continuous tracking and surveillance of health status did not exist thus far, means for patients to report on their current state had to be integrated. Another particularity was the progressive paralysis of patients. Due to impaired fine motor skills, it was difficult for these patients to use commonly available user interfaces such as mouse or multi touch devices. Hence, in view of accounting for the patients' physical state, the NFC-based solution was preferred. Our concept of inSERT, an easy-to-use electronic data capture system for patients with impaired fine motor skills, is based on the following main goals: 
- Increase the usability (a) ease of use \& (b) perceived usefulness: A major problem in mobile user-interface (UI) design practice is that current approaches cannot directly be applied to mobile phones. According to Subramanya et al. (2006), in general, the UI should be intuitive and easy to use. The usability is important for creating applications which are easy to use and which fulfill the needs of the targeted user-group. Ease of use and perceived usefulness derive directly from the TAM (Davis et al., 1989).

- Increase intention to use: The dimension intention to use is a predictor of future usage, it derives directly from the TAM (Davis et al., 1989).

- Increase confirmation: The questions about confirmation are an important dimension for explaining IS continuance intention (Bhattacherjee, 2001).

- Providing a very efficient and intuitive way of interaction suited to patients with impaired fine motor skills: It is important that patients with impaired fine motor skills are able to use the system. Otherwise, it is no guarantee that the data will be entered reliably.

- Improve information quality: Are the results useful to serve as medical decision-making basis and facilitate the additional information of physician daily work?

\subsection{Research Approach}

We used a design science approach (Jones and Gregor, 2007, Hevner et al., 2004) which aims to develop solutions to organizational problems through design and evaluation of novel artefacts. The design process generally consists of analyses, design, implementation and evaluation of an artefact (Simon, 1996, Hevner et al., 2004), in our case the prototype inSERT. As users generally had no prior experience with mobile EDC services, and user involvement in system development is still an acknowledged success factor, we adopted the AAL service design approach (AALSDA) approach by (Menschner et al., 2011).

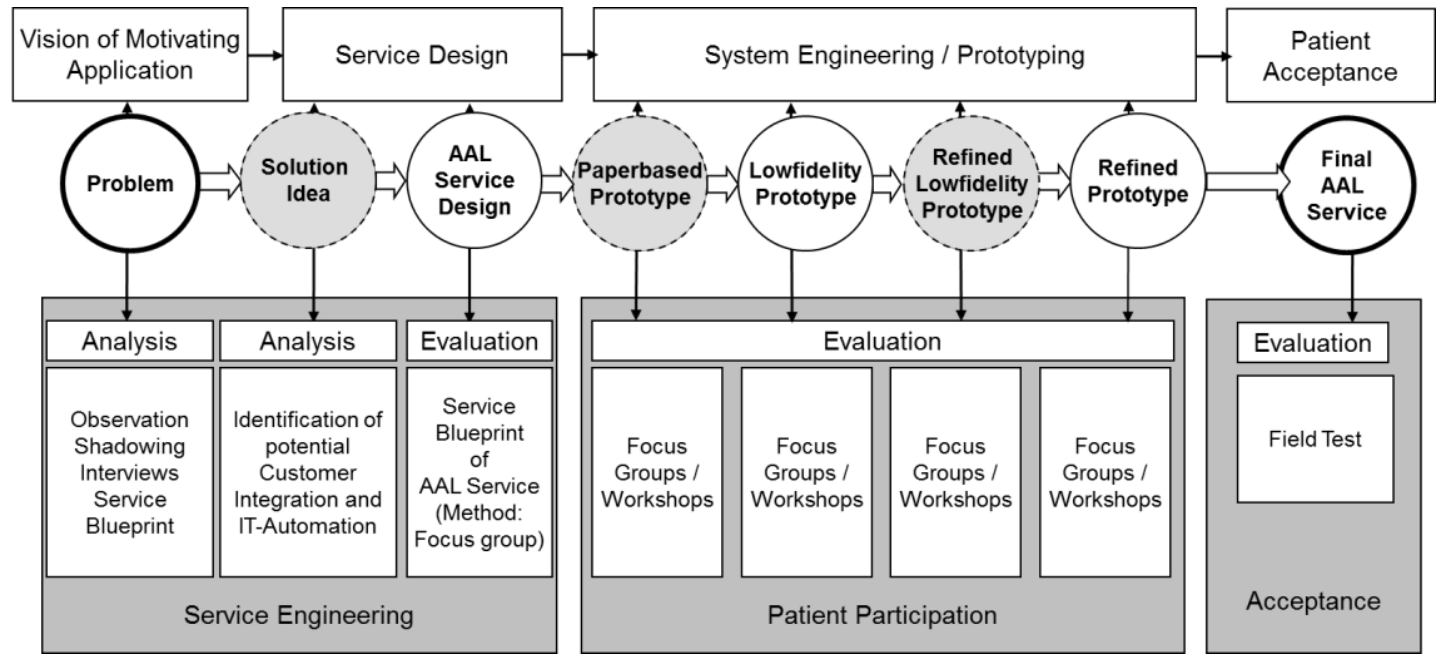

Figure 1. AALSDA approach (Menschner et al., 2011).

We began with an in-depth analysis of treatment and care processes of patients suffering from ALS. Background information on the problem was obtained through a review of relevant literature, case studies, interviews, questionnaires, observations and document analyses. Based on the findings from the analyses and our design goals, we developed a design concept and a low fidelity prototype which was then evaluated in focus groups as well as in workshops (Prinz et al., 2011). We then developed a fully functional proof-of-concept prototype which was tested in a long-term field study in a German hospital. 


\section{The Design of the inSERT Prototype}

A mobile phone application that allows the reading of NFC-tags on a smart poster with NFC-enabled mobile phones is used. By touching a tag with an NFC-enabled mobile phone, this information can be read and displayed to the user (Forum, 2006). The transmitted and aggregated data is processed and stored in the central unit of the inSERT system and can be analyzed by physicians and nurses.

\subsection{Smart Poster and Mobile Application}

In the tests we executed during the design process, we show that the NFC solution outperforms iPhone and PC-solutions as well as a paper-based protocol with respect to the following criteria on pragmatic and hedonistic aspects (Prinz et al., 2012). The easy to learn touch metaphor of NFC interaction makes NFC, particularly for the target group (elderly people and people with impaired fine motor skills) who are not familiar with the use of mobile phones or computers, an interesting technology.

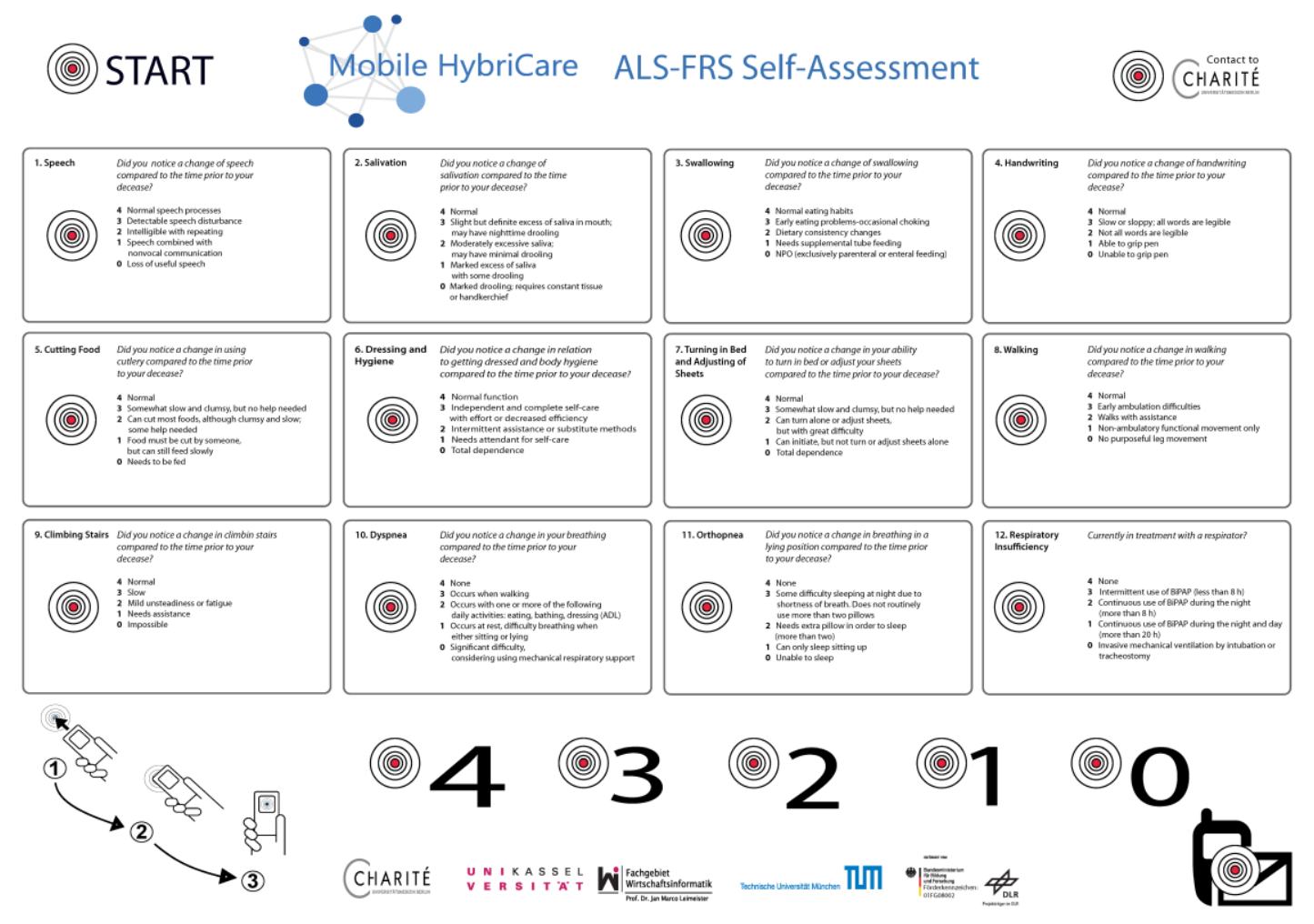

Figure 2. $\quad$ NFC Smart poster for patient self-reporting

Figure 2 shows the self-rating questionnaire smart poster of the well-established ALS-functional rating scale (Kaufmann et al., 2005). The smart poster contains twelve questions (three sections to bulbar symptoms, motor activity and respiratory dysfunction) with five possible answers in each case (Kasarskis et al., 1999). The poster has images printed in front and NFC-tags glued to the back.

To start the application, a touch on the "start" icon or interaction field is sufficient. After the application is launched, the patient has to touch one of the twelve questions. As shown in figure 3, patients have to follow four simple steps to answer the question via inSERT. 
1. Touch the "start" icon to launch the application installed on the phone.

2. Touch one of the twelve questions.

3. Touch again on the rating scale to answer the question.
3. (Step two and three is repeated twelve times)

4. Touch on the "senden" [send] tag and confirm it with a second touch to send the data to the physician.

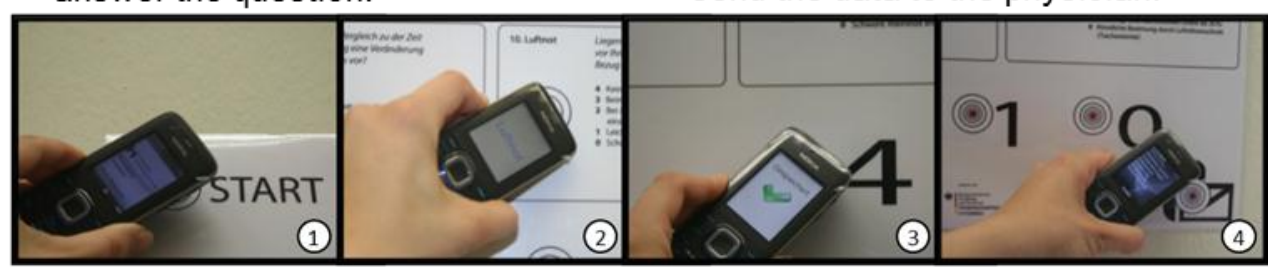

Figure 3. Rating process of inSERT

Once contact is made, the mobile device gives audible, haptic and visual feedback to the user. For audible feedback, the title of the chosen question is spoken; for haptic feedback, the phone vibrates; and for visual feedback, the chosen headline of the question appears on the display. After each question the patient has to respond to a five-point Likert scale (0 to 4). Once the answering process for the chosen question is completed, the application reminds the patient to answer the next question. Again, the mobile device confirms the transmission of data with audible, haptic and visual feedback.

The icon of the interaction field is based on generic NFC tags (Arnall, 2006). To help patients navigate to the interactive areas, the inner circle is coloured red. The size of the interaction field of $35 \times 35$ millimetres is dependent on the used passive NFC tags.

The client is implemented as a Java J2ME midlet which controls the NFC-reader device of the Nokia 6212 phone through an API provided by Nokia. J2ME is an optimized Java Edition for programming mobile devices (Java, 2010). The multimedia files, such as photos and audio data corresponding to the twelve questions, are stored on the mobile device. In order to start the application, an NFC-Tag, storing a specific NFC Data Exchange Format (NDEF) record-type is used. The URN can be assigned to a Java class in the mobile phone. If the mobile device detects an NFC tag with the stored URN, the mobile phone selects the assigned java class and executes it. If the patient wants to send a message to the hospital, he can use the email tag which prompts the midlet to generate a special enquiry to the server.

\subsection{Physician Dashboard}

To allow physicians the management of the patient scoring data, a so-called "physician dashboard" has been implemented through which physicians can extract relevant information about their patients. In order for the information to be available anytime and anywhere, a web portal has been implemented for access by both stationary computers and smart phones. The dashboard is structured as follows: On one side there is a category 'patients' providing patient relevant data. On the opposite side there is a category 'mobile devices' referring to the mobile phone data handed out. In the 'patients' category, physicians get an overview representation of the patient data, where they can see information about all participating patients. This is where patients can be added or deleted. Next to the personal data, such as name and date of birth, is the place where the ID assigned to each patient can be found. This ID is also provided on the smart phone to uniquely and anonymously identify a patient. In addition to the ID, the data referring to the responsible physicians can be obtained. The display can be customised as a table or as a figure, depending on the user's preferences. 


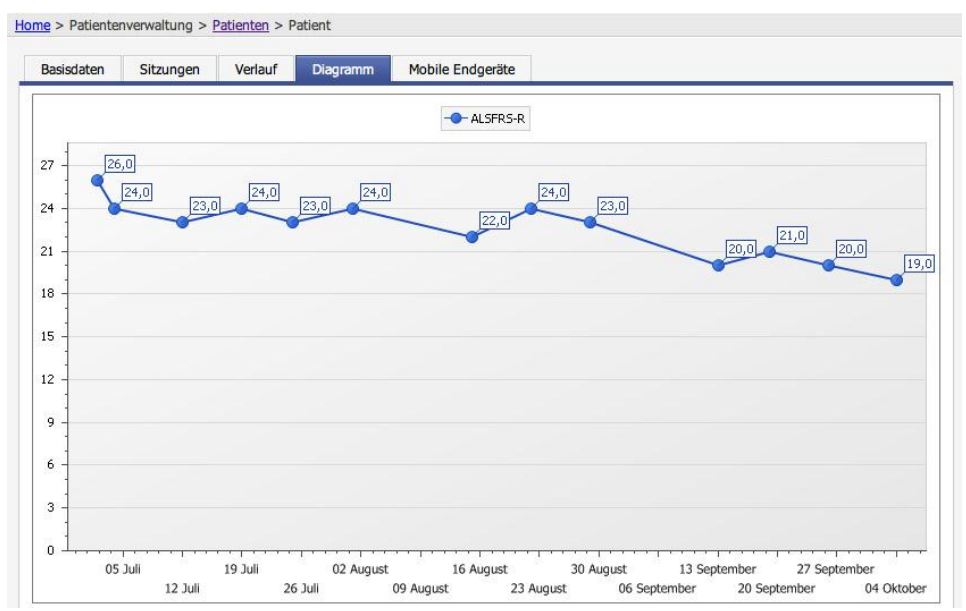

Figure 4. $\quad$ Physicians dashboard: Patient Scoring Data

Figure 4 depicts the answers of the last questionnaire entries. The diagram clearly illustrates the changes in the assessment of patients over their period of scoring. In addition to the content preparation for patients, the IDs assigned to dedicated patients in the category 'mobile devices' can be edited. Thus, it is possible to allot a certain beginning and end point for when the ID can be used.

In addition to the access for physicians, the dashboard contains a special administrator access. This area allows assigning of access privileges to specific people in different roles. It is thus possible to define the physicians responsible for patients or further administrators can be allotted. The administrator access also allows adding additional questionnaires. Therefore, a part from the existing system of evaluating a questionnaire concerning motor diseases, further smart poster and tele-medical applications can be appended following similar principles.

\subsection{Technical Description and Process Routine}

To gather data in a centralised way and to easily monitor the data, a client server architecture is chosen. In this architecture, the mobile phone acts as a client gathering the data and passing it on to a server. The choice of client application, rather than purely server-side architecture, is based on the fact that, especially in rural areas, mobile communication networks are insufficiently developed for broadband connection, needed to exchange intensive multimedia data between the server and mobile devices (BMWi, 2009). The client software is implemented on a Nokia 6212 classic NFC-enabled mobile phone.

The data-flow from mobile device to the server can be detailed as follows:

- By touching a tag, the inSERT midlet application is automatically started, and the mobile receives the identifier of the poster. The mobile device then requests an enquiry to the server. This enquiry incorporates the Poster-ID as well as a unique hash value.

- The server matches the hash value with the ones issued to the patients. If the hash value exists, it sends the tag-IDs available on the poster to the mobile phone. This gives the phone the semantics of which tag corresponds to what meaning.

- The mobile phone sends the data to the server once the patient completes the evaluation and touches the send-tag twice.

- In this implementation, the intermediary server is first being addressed, and it eventually composes the respective messages using scripting and Java servlets.

In the hospital, the transmitted data is merged with the patient data using the delivered ID. The central unit of the electronic data capture solution is a server which stores the patients' response to the questionnaire and can be accessed through a web interface. Since the user data contain sensitive information about patients, data security has to be considered. This is achieved in the following way: There 
is separation between the master data and transaction data. Transaction data is delivered by secured mechanisms to the hospital. However, master data (in the form of information about the patients) is located in a shielded domain in a secured area inside the server architecture.

\section{Evaluation Setting}

The prototype was evaluated in a field study at patients' home with 18 patients' (amongst them 7 female), ranging in age from 55 to 70 years, of a German hospital over the course of 12 weeks. First, the physicians received a 45 minutes hands-on training to use and explain the system. After that, the physicians gave a training session to the patients, so that the patients were able to use the system at home. Patients received a fixed day of the week on which they should conduct their health status with the prototype. The hospital provided patient access for us, yet they took over the distribution process of the NFC-enabled mobile phones and smart posters. We had two drop outs, one of them died during the field test, the other one had usage problems - the power on/off button of the mobile phone was too small.

The objectives of this field study were (1) a test of the technical feasibility and (2) an evaluation of the practicability, according to our design goals.

- Test of the technical feasibility of the NFC-based electronic data capture system for patients with impaired fine motor skills: The relevant issue in this case was to find out whetherthe patients are able to use the NFC-based mobile phones with the smart poster with an acceptable error rate.

- Evaluations of the practicability of the electronic data capture system: In order for the proposed system to be beneficial in capturing patient data, the system would have to be able to run stable at patients' home without attendance, aside from assembly and disassembly. Furthermore, it needs to be assessed whether the prototype application was capable of gathering patient data at patients' home.

Every participant received an NFC-enabled mobile phone and a smart poster containing twelve questions about the level of functional impairment in performing one of twelve common tasks, e.g. climbing stairs, speaking or breathing. Each task is rated on a five-point scale from $0=$ can't do, to $4=$ normal ability. The patient has to rate him- or herself once a week on a fixed day. This poster represents the ALS-FRS rating scale, which is well-established for ALS-patients (Kaufmann et al., 2005). After twelve weeks, the patients fill in a questionnaire (regarding usability, intention to use, confirmation \& interaction) with a 7-point likert scale and post it together with the mobile phone and smart poster in a prepaid package to us. We received all distributed mobile phones and smart posters, but only 13 of 18 questionnaires.

\section{Evaluation Results}

The results of the evaluation are structured using the design goals (1) increasing usability, (2) intention to use, (3) confirmation, (4) providing a very efficient and intuitive way of interaction suited to patients with impaired fine motor skills and (5) improving information quality. Interpretations are accompanied by information gathered during telephone interviews with patients and physicians. We conducted evaluations on a technological (technical feasibility), user (practicability) and physician's basis.

\subsection{Overall Impression}

The evaluation shows that the prototype has great potential to improve the information logistics problem between physicians, home care nurses, and patients with impairment of fine motor skills. Through the active participation and integration of patients, better documentation and an improved basis for medical treatment and nursing care could be achieved. Further, through an intensified integration, pa- 
tients were enabled to better understand their medical condition and could participate more actively in the treatment and consultation processes. Our results indicate that customer (in our case patients) integration can lead to modifications for an enhanced patient-physician relationship enabled by improved patient information and autonomy. We also surmise that the prototype can improve productivity of medical processes not only by accounting for improved standards of medical care and quality of life, but also by being more cost efficient. "The use of inSERT is almost as simple as filling in a paperbased questionnaire!" One of the patients died during the field study; with the help of the captured data this could have been anticipated by a physician shortly before. Generally, a healthy person starts with an ALSFRS base score of 48. Our patients had an average score of 31,15 points, starting with an average of 33,72 and finished after twelve weeks with an average of 30,61. If the score is less than 20 points, the patient will normally die within the next few weeks. The mentioned patient started with a score of 24 and fell below the threshold of 20 within a few weeks. Furthermore, we received requests from three patients whether they could continue to use the system even after the end of the field test. The physicians' feedback was positive and there were no complaints. The subjectively assessed values have been regarded as realistic by the physicians. They checked the transmitted data with values before and after the field test. "With the help of the transmitted data, we have an accurate data base on the individual patient. Before we used the system, we have examined the patient every three to six months and in the meantime we have received no information about the patients' health status. The additional data allows us to adjust the treatment if the patient's condition changes."

\subsection{Evaluation according to design goals}

The results of the evaluation are structured according to the five design goals. The average values of the individual items can be found in table 1 .

- Increase the usability (1a) ease of use \& (1b) perceived usefulness: The overall feedback of the user acceptance is very high. Thus, it can be assumed that the operation with the prototype is easy to learn and comprehend. The patients scored the used EDC system as a system which is easy to use for them. The interaction with the system did not require a lot of mental effort. The perceived usefulness has been rated as high, with the use of inSERT the documentation frequency of disease progression would increase. Furthermore, the usefulness for documenting the disease progression has been rated high. The patients could use the system without the help of relatives or nursing staff.

- Increase intention to use (2): As we can see in the evaluation results, the intention to use has been rated high. The results match the statements of telephone interviews and showed that some patients are interested in keeping the system in use after the field study.

- Increase confirmation (3): The confirmation of the system has been rated high. The functionalities of the system were better than the patients had expected. Overall, the expectations of the system were fulfilled. In summary, we could say that all the basic features were present and the patients did not miss any main functionality.

- Providing a very efficient and intuitive way of interaction suited to patients with impaired fine motor skills (4): With inSERT we can show, that the system provides a very efficient and intuitive way of interaction suited to patients with impaired fine motor skills. The patients have rated the use of NFC technology very high, the use of buttons to interact with the system scored worse. The vibration and acoustic statement of the mobile phone by touching an interactive field was a helpful functionality.

- Improve information quality (5): As a test of the technical feasibility of the systems, the mentioned field study was a great success. We received 10.7778 (average) records per patients with a standard deviation of 3.6871. A total of 2328 values were transmitted, with an error rate of 0.34 percent ( 8 data points), which have been caused by a double assessment or missing data point. The average assessment interval was 176:40:24 hours with a standard deviation of 64:23:41 hours. With the collected data, the physicians have a weekly picture of the patient's condition. Before they used the system, they generated this data only when the patients came to the hospital during regular appointments every three to six months. 


\begin{tabular}{|c|c|c|c|c|}
\hline Goal & $\begin{array}{l}\text { Variable } \\
\text { (Source) }\end{array}$ & Measurement Items (Range 1 to 7 ) & AVG & SD \\
\hline \multirow[t]{3}{*}{ (1a) } & \multirow{3}{*}{$\begin{array}{l}\text { Ease of use } \\
\text { (Davis et } \\
\text { al., 1989) }\end{array}$} & Learning to operate with inSERT is easy for me. & 6.77 & .439 \\
\hline & & Interacting with inSERT requires a lot of mental effort. & 1.77 & 1.691 \\
\hline & & I find inSERT easy to use. & 6.62 & .506 \\
\hline \multirow[t]{2}{*}{ (1b) } & \multirow{2}{*}{$\begin{array}{l}\text { Perceived } \\
\text { usefulness } \\
\text { (Davis et } \\
\text { al., 1989) }\end{array}$} & $\begin{array}{l}\text { Using inSERT would increase the frequency of documentation of } \\
\text { disease progression. }\end{array}$ & 6.5 & .972 \\
\hline & & $\begin{array}{l}\text { I find inSERT to be useful for documenting my disease progres- } \\
\text { sion. }\end{array}$ & 6.7 & 675 \\
\hline \multirow[t]{2}{*}{2} & \multirow{2}{*}{$\begin{array}{l}\text { Intention } \\
\text { to use } \\
\text { (Davis et } \\
\text { al., 1989) }\end{array}$} & Assuming that I have access to inSERT, I intend to use it. & 5.09 & 1.287 \\
\hline & & Given that INSERT is available to me, I predict that I will use it. & 6.09 & 1.375 \\
\hline \multirow[t]{2}{*}{3} & \multirow{2}{*}{$\begin{array}{l}\text { Confirma- } \\
\text { tion } \\
\text { (Bhattache } \\
\text { rjee, 2001) }\end{array}$} & $\begin{array}{l}\text { The functionality provided by inSERT was better than what I had } \\
\text { expected. }\end{array}$ & 5.83 & 1,899 \\
\hline & & $\begin{array}{l}\text { Overall, most of my expectations of using inSERT were con- } \\
\text { firmed. }\end{array}$ & 6.23 & 1.166 \\
\hline \multirow[t]{4}{*}{4} & \multirow{4}{*}{$\begin{array}{l}\text { Intuitive } \\
\text { way of } \\
\text { interaction }\end{array}$} & $\begin{array}{l}\text { I prefer the use of inSERT via NFC (touching interactive fields on } \\
\text { the poster) }\end{array}$ & 6.55 & 1.036 \\
\hline & & $\begin{array}{l}\text { I prefer the use of inSERT via button (without touching interac- } \\
\text { tive fields on the poster). }\end{array}$ & 2.82 & 1.136 \\
\hline & & $\begin{array}{l}\text { The acoustic statement of each question section was helpful func- } \\
\text { tionality. }\end{array}$ & 6.67 & .888 \\
\hline & & $\begin{array}{l}\text { The vibration of the mobile phone by touching a question has } \\
\text { been a helpful functionality. }\end{array}$ & 6.33 & 1.723 \\
\hline
\end{tabular}

Table 1.

Evaluation results of inSERT

The prototype further revealed some design principles for NFC-based EDC in general. These include the importance of feedback (audible, visible and haptic), a comprehensible and coherent poster structure and measurements to ensure data security. Noted was the appreciation of the automated reminders and warnings. Patient responses in our study showed that this gave them a feeling of being cared for. We assume that the prototype system could be applied to other contexts or groups of patients in health care, such as elderly people who face similar difficulties in handling electronic devices.

\section{Conclusion and future work}

Because of the rarity of the disease, we only had a very small number of cases and a generalisation is not always possible. This research paper describes the development, implementation and evaluation of an application that allows patients with impaired fine motor skills to assess their health status, based on an established rating scale. We therefore adapted NFC technology to a quality of life questionnaire. We focused on engineering an easy to use interaction. Our evaluation showed that patients with impaired fine motor skills accepted inSERT as a system that is easy to use and effective for reporting the patient's current condition. Through the active participation and integration of patients, improved documentation as well as an improved basis for medical treatment and nursing care could be achieved. Further, through an intensified integration, patients gained better understanding of their medical condition and were able to participate more actively and cooperate in the treatment and consultation processes. The example of inSERT shows the potentials and possibilities that technical support systems can offer to patients. The latter exemplary extension is based upon literature documenting that the completion of specific questionnaires enable improved forecasts of disease progression (Kaufmann et al., 2005). The recent integration of sensors (e.g., motion sensor) and additional technical devices (e.g., global positioning system (GPS) devices) in new generations of mobile phones additionally enable completely novel medical applications (Schweiger et al., 2007), which could extend our work. To 
conclude, the use of mobile services and information technology can have a great impact on medical processes and services. Improvements of communication and interaction processes have a positive influence on the quality of patients' lives. A modified patient-physician relationship, as a result of improved patient information and autonomy, offers new possibilities for the design of novel medical services which are only made possible by modern technical support systems.

\section{References}

Arnall, T. (2006). A graphic language for touch-based interactions. 8th conference on Humancomputer interaction with mobile devices and services Helsinki, Finland: ACM.

Bhattacherjee, A. (2001). Understanding Information Systems Continuance: An ExpectationConfirmation Model. MIS Quarterly, 25 (3), 351-370.

Bischoff-Ferrari HA., Vondechend M., Bellamy N. and R., T. (2005). Validation and patient acceptance of a computer touch screen version of the WOMAC 3.1 Osteoarthritis Index. Ann Rheum Dis., 64 (1), 80-84.

Blake, H. (2008). Innovation in practice: mobile phone technology in patient care. Br J Community Nurs., 13 (4), 160, 162-165.

BMWi (2009). Breitbandstrategie der Bundesregierung. Berlin: Bundesministerium für Wirtschaft und Technologie (BMWi).

Bravo, J., López-de-Ipiña, D., Fuentes, C., Hervás, R., Peña, R., Vergara, M. and Casero, G. (2008). Enabling NFC Technology for Supporting Chronic Diseases: A Proposal for Alzheimer Caregivers. In Ambient Intelligence. (Aarts, E., Crowley, J., De Ruyter, B., Gerhäuser, H., Pflaum, A., Schmidt, J. and Wichert, R. Eds.), 109-125, Springer Berlin / Heidelberg.

Dale O. and KB., H. (2007). Despite technical problems personal digital assistants outperform pen and paper when collecting patient diary data. J Clin Epidemiol, 60, 8-17.

Davis, F. D., Bagozzi, R. P. and Warshaw, P. R. (1989). User acceptance of computer technology: a comparison of two theoretical models. Manage. Sci., 35 (8), 982-1003.

ECMA-340 (2004). Near Field Communication Interface and Protocol (NFCIP-1).

Fikry, M., Karim, A. and Muhamad, R. (2006). Integration of Near Field Communication (NFC) and Bluetooth Technology for Medical Data Acquisition System Computational Geometry \& Artificial Vision, 147-152.

Forum, N. (2006). Smart Poster Record Type Definition. Available: http://www.nfc-forum.org/specs/ [Accessed 18.10 2010]

Grauer, H. and Birnbom, F. (1975). A Geriatric Functional Rating Scale to determine the need for institutional care. J Am Geriatrics Soc, 23, 472-476.

Guyatt, G. H., Feeny, D. H. and Patrick, D. L. (1993). Measuring health-related quality of life. Ann Intern Med, 118 (8), 622-629.

Hevner, A. R., March, S. T. and Park, J. (2004). Design science in information systems research. Management Information Systems Quarterly, 28 (1), 75-105.

Iglesias, R., Parra, J., Cruces, C. and Segura, N. G. d. (2009). Experiencing NFC-based touch for home healthcare. Proceedings of the 2nd International Conference on Pervasive Technologies Related to Assistive Environments. Corfu, Greece: ACM.

Java (2010). What is J2ME or Java ME? . Available: http://www.java.com/en/download/faq/whatis j2me.xml [Accessed 20.08 2010]

Jones, D. and Gregor, S. (2007). The Anatomy of a Design Theory. Journal of the Association for Information Systems, 8 (5), 312-335.

Kasarskis, E. J., Scarlata, D., Hill, R., Fuller, C., Stambler, N. and Cedarbaum, J. M. (1999). A retrospective study of percutaneous endoscopic gastrostomy in ALS patients during the BDNF and CNTF trials. Journal of the Neurological Sciences, 169 (1-2), 118-125.

Kaufmann, P., Levy, G., Thompson, J. L. P., DelBene, M. L., Battista, V., Gordon, P. H., Rowland, L. P., Levin, B. and Mitsumoto, H. (2005). The ALSFRSr predicts survival time in an ALS clinic population. Neurology, 64, 38-43. 
Koene, P., Köbler, F., Burgner, P., Resatsch, F., Sandner, U., Leimeister, J. M. and Krcmar, H. (2010). RFID-based media usage panels in supportive environments. 18th European Conference on Information Systems (ECIS). Pretoria, South Africa.

Lahtela, A., Hassinen, M. and Jylha, V. (2008). RFID and NFC in healthcare: Safety of hospitals medication care. 2008 Second International Conference on Pervasive Computing Technologies for Healthcare. Tampere, Finland.

Leimeister, J. M., Krcmar, H., Horsch, A. and Kuhn, K. (2005). Mobile IT-Systeme im Gesundheitswesen, mobile Systeme für Patienten. HMD Praxis der Wirtschaftsinformatik, 41 (244), 74-85.

Löser, C., Lübbers, H., Mahlke, R. and Lankisch, P. G. (2007). Der ungewollte Gewichtsverlust des alten Menschen. Dtsch Arztebl, 104 (49), 3411-3420.

Menschner, P., Prinz, A., Koene, P., Köbler, F., Altmann, M., Krcmar, H. and Leimeister, J. (2011). Reaching into patients' homes - participatory designed AAL services. Electronic Markets, 1-14.

Morak, J., Hayn, D., Kastner, P., Drobics, M. and Schreier, G. (2009). Near Field Communication Technology as the Key for Data Acquisition in Clinical Research. First International Workshop on Near Field Communication. Hagenberg, Austria.

Morak, J., Kollmann, A., Hayn, D., Kastner, P., Humer, G. and Schreier, G. (2007). Improving telemonitoring of heart failure patients with NFC technology. Proceedings of the fifth IASTED International Conference: biomedical engineering. Innsbruck, Austria.

Nyholm, D., Kowalski, J. and Aquilonius, S.-M. (2004). Wireless real-time electronic data capture for self-assessment of motor function and quality of life in Parkinson's disease. Movement Disorders, 19 (4), 446-451.

Palmblad M. and B., T. (2004). Electronic diaries and questionnaires: designing user interfaces that are easy for all patients to use. Qual Life Res., 13, 1199-1207.

Prinz, A., Menschner, P., Altmann, M. and Leimeister, J. M. (2011). inSERT - an NFC-based SelfReporting Questionnaire for Patients with Fine Motor Diseases. 3rd International Workshop on Near Field Communication - NFC2011 Hagenberg.

Prinz, A., Menschner, P. and Leimeister, J. M. (2009). NFC-basiertes Ernährungsmanagement für ältere, pflegebedürftige Menschen. Informatik 2009 - Im Focus das Leben. Jahrestagung der Gesellschaft für Informatik. Lübeck: GI - Gesellschaft für Informatik, GI Lecture Notes in Informatics.

Prinz, A., Menschner, P. and Leimeister, J. M. (2012). Has NFC the Potential to Revolutionize Selfreported Electronic Data Capture? - An Empirical Comparison of Different Interaction Concepts CHI Conference on Human Factors in Computing Systems. Austin, USA: ACM.

Richter, J. G., Nixdorf, M., Koch, T., Schneider, M., Becker, A. and Monser, R. (2006). Mobile Computing instead of paper based documentation in German Rheumatology. Proceedings of the International Conference on Mobile Business IEEE Computer Society.

Schweiger, A., Sunyaev, A., Leimeister, J. M. and Krcmar, H. (2007). Toward seamless healthcare with software agents. Communications of the Association for Information Systems (CAIS), 19, 692-709.

Simon, H. A. 1996. The Sciences of the Artificial (3rd ed.), Cambridge, MA, MIT Press.

Strömgren, A. S., Groenvold, M., Sorensen, A. and Andersen, L. (2001). Symptom recognition in advanced cancer. A comparison of nursing records against patient self-rating. Acta Anaesthesiologica Scandinavica, 45 (9), 1080-1085.

Subramanya, S. R. and Yi, B. K. (2006). User interfaces for mobile content. Computer, 39 (4), 85-87.

Velikova, G., Wright, E. P., Smith, A. B., Cull, A., Gould, A., Forman, D., Perren, T., Stead, M., Brown, J. and Selby, P. J. (1999). Automated Collection of Quality-of-Life Data: A Comparison of Paper and Computer Touch-Screen Questionnaires. J Clin Oncol, 17 (3), 998-1007.

Want, R. (2006). An Introduction to RFID Technology Pervasive Computing, 6, 25-33.

Welsh, M., McDermott, M. P., Holloway, R. G., Plumb, S., Pfeiffer, R. and Hubble, J. (2003). Development and testing of the Parkinson's disease quality of life scale. Movement Disorders, 18 (6), 637-645.

Wolfe, F. and Pincus, T. (1995). Data collection in the clinic. Rheumatic Disease Clinics of North America, 21 (2), 321-358. 